\title{
A Computational Framework for Transmission Risk Assessment of Aerosolized Particles in Classrooms
}

\author{
Kendrick Tan \\ lowa State University \\ Boshun Gao \\ lowa State University \\ Cheng-Hau Yang \\ lowa State University \\ Emily Johnson \\ lowa State University \\ Ming-Chen Hsu \\ lowa State University \\ Alberto Passalacqua \\ lowa State University \\ Adarsh Krishnamurthy \\ lowa State University \\ Baskar Ganapathysubramanian ( $\sim$ baskarg@iastate.edu ) \\ lowa State University
}

\section{Research Article}

Keywords: Risk Assessment, Aerosolized Particulate Transfer, CFD, Classroom

Posted Date: September 24th, 2021

DOI: https://doi.org/10.21203/rs.3.rs-902643/v1

License: (c) (i) This work is licensed under a Creative Commons Attribution 4.0 International License.

Read Full License 


\title{
A Computational FrameWORK FOR TRANSMISSION Risk Assessment of Aerosolized Particles in Classrooms
}

\author{
Kendrick Tan ${ }^{1}$, Boshun Gao ${ }^{1}$, Cheng-Hau Yang ${ }^{1}$, Emily L. Johnson ${ }^{1}$, Ming-Chen Hsu ${ }^{1}$, \\ Alberto Passalacqua ${ }^{1}$, Adarsh Krishnamurthy ${ }^{1}$, Baskar Ganapathysubramanian ${ }^{1 *}$ \\ ${ }^{1}$ Mechanical Engineering \\ lowa State University \\ * Corresponding Author Email: baskarg@iastate.edu
}

\begin{abstract}
The ongoing COVID-19 pandemic has rendered confined spaces as high-risk areas. There is an increasing push to resume in-person activities, for instance, teaching in K-12 and university settings. It becomes important to evaluate the risk of airborne disease transmission while accounting for the physical presence of humans, furniture, and electronic equipment, as well as ventilation. Here, we present a computational framework based on detailed flow physics simulations that allows straightforward evaluation of various seating and operating scenarios to identify risk factors and assess the effectiveness of various mitigation strategies. These scenarios include seating arrangement changes, presence/absence of computer screens, ventilation rate changes, and presence/absence of mask-wearing. This approach democratizes risk assessment by automating a key bottleneck in simulation-based analysis-creating an adequately refined mesh around multiple complex geometries. Not surprisingly, we find that wearing masks (with at least $74 \%$ inward protection efficiency) significantly reduced transmission risk against unmasked and infected individuals. The availability of such an analysis approach will allow education administrators, government officials (courthouses, police stations), and hospital administrators to make informed decisions on seating arrangements and operating procedures.
\end{abstract}

\section{Keywords}

Risk Assessment | Aerosolized Particulate Transfer | CFD | Classroom

\section{Introduction}

The SARS-CoV-2 virus pandemic has irreversibly changed how we consider transmission risk in indoor environments. The physical distancing or six-foot rule 112 , for instance, is a guideline that does not account for small aerosol droplets that are continuously mixed through an indoor space. This is especially alarming considering that airborne transmission-via small aerosolized particles-of COVID-19 is widely recognized ${ }^{3-6}$. The distribution of aerosolized particles depends on a dizzying array of factors, including ventilation and air filtration rates, airflow patterns in the indoor space that are impacted by furniture, thermal fluxes on the room facade, and respiratory activity of the inhabitants. While tools for risk assessment have recently been developed ${ }^{7+9}$, most such tools make significant assumptions on the indoor environment (well-mixed air, no thermal plumes from equipment and occupants) and the respiratory activity (exhalation of virion particles). Thus, while conventional tools may indicate that a particular room is low-risk on average, there may be specific locations in the room with significantly higher risk for transmissionfor example, in areas where local recirculation causes limited air exchange with the outside environment. Location-specific risk assessment becomes especially important if individuals are seated in such locations for extended periods, increasing their cumulative exposure time, such as our K-12 students, public service workers, and essential workers. For instance, courthouse activities require participants (judges, clerks, petitioners, jurors) to remain sedentary over long periods. Similarly, most classroom activities require students to remain seated for extended periods. In such scenarios, it becomes imperative to identify if specific locations have higher risk and rank among alternate arrangements.

Consider the case of aerosols (with particles $<10 \mu \mathrm{m}$, which exhibit significantly larger traveling distances 10 . These respiratory particles are suspended in a warm and moist puff cloud, which increases the traveling distance of the respiratory particles before they settle onto surfaces due to gravity 11 . The additional distance traveled by small respiratory droplets can also be attributed to expired jets (even with the use of face 
masks) ${ }^{12}$. The transport of the small respiratory particles within an enclosed space may also be complicated by the flow field induced by ventilation systems and the thermal plumes from computers and human occupants 13 . The tendency of droplets to remain airborne may be further affected by ambient flow characteristics such as the flow speed, turbulence intensity, direction, temperature, and relative humidity 14 . Apart from their potential to become airborne, small respiratory particles also can penetrate deeper into the human lower respiratory tract ${ }^{15}$ and have higher infectivity than larger cough droplets ${ }^{16}$. Maintaining a physical distance from an infected individual does not entirely protect susceptible individuals from inhaling enough respiratory aerosols to cause infection 14 . Hence, there is a pressing need to accurately model the transport of virion-laden aerosols, especially in indoor settings, to identify (and mitigate) risk zones.

In this context, high-fidelity fluid simulations provide a practical approach to evaluate various seating and operating scenarios, identify risk factors, and assess mitigation strategies. Ideally, such approaches need to be rapidly deployable, customizable to specific scenarios (geometry, occupant conditions, environmental conditions, ventilation), and suitable for extracting reliable best practices. A major challenge in this type of simulation is the mesh generation step. The resolution and quality of the mesh are intimately related to the overall accuracy of the simulation. Although mesh generation is a fundamental part of numerical approaches, creating high-quality meshes continues to be a significant bottleneck in the overall workflow. This restriction is exacerbated when considering adaptivity and becomes exceptionally challenging in the presence of an arbitrarily shaped geometric object. Streamlining this workflow is one of the components of the NASA 2030 computational fluid dynamics (CFD) milestone towards the goal of conducting overnight large-eddy simulations (LES) ${ }^{17}$ : "Mesh generation and adaptivity continue to be significant bottlenecks in the CFD workflow."

Here, we deploy a framework that can democratize the execution of such risk assessment studies. This framework consists of (a) immersogeometric carved-out analysis that allows automated consideration of geometrically complex objects in a principled analysis platform 18 , (b) a massively parallel octree-based mesh generator that rapidly constructs high-quality meshes ${ }^{19}$, (c) a detailed flow physics simulation (LES) to produce accurate flow features $\sqrt{20}$, (d) a coupled thermal solver to account for the thermal plumes produced by humans and electronics 21 , and (e) a passive scalar transport model that accounts for the distribution of virion-laden aerosols. We illustrate this framework by assessing the risk of transmission across various seating plans, operating conditions, and aerosol source locations in a canonical classroom setting, specifically a room where several of us teach (Figure 1). The availability of such an analysis approach will allow education administrators (K-12, university), federal/state/local government officials (courthouses, police stations), and hospital administrators to make informed decisions on seating arrangements and operating procedures.

\section{Results}

For the results in this work, we consider a typical university classroom that will be employed to investigate the risk of virus transmission. Figure 1a shows the classroom environment prior to the COVID-19 pandemic. As shown in Figure $1 \mathrm{~b}$ the room has a length of $9 \mathrm{~m}$, a width of $9 \mathrm{~m}$, and a height of $3.5 \mathrm{~m}$ with a ventilation system with eight vents (four inlet vents and four outlet vents) installed on the ceiling. The occupants are modeled using labeled mannequins (Figure 1c).

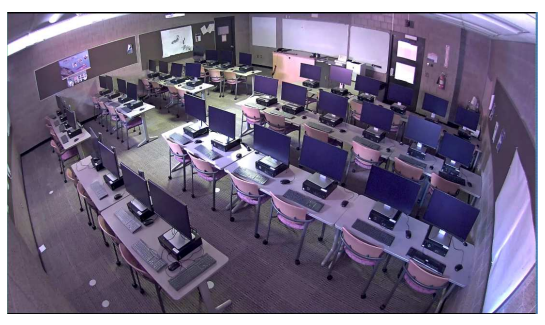

(a) The actual classroom environment

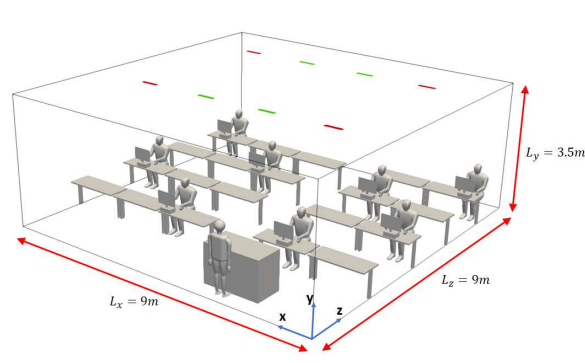

(b) Computational domain with computers

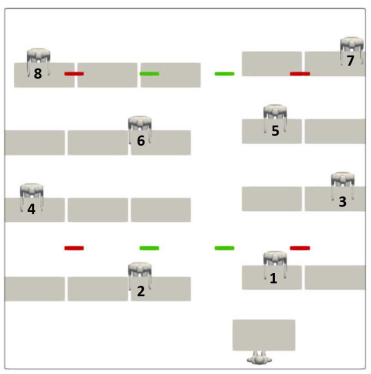

(c) Vents and mannequin labels

Figure 1: Computational representation (b) of the enclosed space (a) to be used in the simulation. Top-down view of the classroom (c) with the inlet (green) and outlet (red) vents on the ceiling. 
We consider several scenarios and evaluate transmission risk. In particular, we consider the following five scenarios: (a) changing seating arrangements to provide six feet of distance between students, which reduces the class size from 32 to 8 students; (b) presence and absence of computer monitors which provide some flow deflection; (c) increased occupancy of the classroom; (d) increased ventilation of six air changes per hour $(\mathrm{ACH})$ compared to the recommended four $\mathrm{ACH}^{22}$; and (e) mask wearing by all versus some versus none of the occupants.

We can assess the transmission risk for the previously mentioned scenarios based on the flow field obtained from the CFD solutions of 3 different configurations (see SI Section C.1). The first configuration (Configuration 1) represents a classroom setting in a lecture setup with 9 occupants (8 seated and 1 standing occupant). The second configuration (Configuration 2) investigates a classroom setting with the same 9 occupants as in Configuration 1, but with an additional computer in front of each seated occupant. The last configuration (Configuration 3 ) includes 21 occupants (20 seated and 1 standing occupant).

For each of these configurations, we automatically create a well-refined mesh and perform combined heat and flow simulations to get the statistically steady flow field (see SI Section A). Then, we consider a coughing event and perform passive scalar transport of virion concentration in the confined space (see SI Section B). We perform this scalar transport for one full air change. Finally, we evaluate transmission risk by considering the inhaled viral load at various spatial locations in the room.

Flow field within the classroom: The flow field within the classroom is primarily influenced by inflow from the inlet vents and the thermal plumes caused by the mannequins and computers. These heated bodies each generate a thermal plume that drives flow upward due to buoyancy. The plume reach is nearly the height of the classroom ( $3.5 \mathrm{~m}$ ), and it creates localized vertical flow features toward the ceiling (Figure 2).

Figure $2 \mathrm{~d}$ shows the streamlines along a vertical slice, the velocity magnitude of the thermal plume generated by a computer $(0.6 \mathrm{~m} / \mathrm{s})$ is found to be approximately twice that generated by a human $(0.35 \mathrm{~m} / \mathrm{s})$. When these plumes reach the ceiling, the flow moves along the ceiling plane, and part of this flow is recirculated back down when it hits the flow emanating from the inlet vents. This recirculation of air within the classroom due to the thermal plumes and inlet jets dilutes the viral load concentration of the contaminated air within the enclosed space. The diluted viral load concentration is eventually removed from the classroom through the outlet vents.

Viral load concentration field: Upon the expulsion of viral-laden aerosols due to a coughing event, we find that the contaminated air eventually gets transported upward due to the thermal plume (see Figure S.9 and Figure S.10). Following the streamlines, the viral load concentration field is recirculated due to the upward motion of the thermal plumes and the downward motion of the inlet flow. Successive recirculation further dilutes the viral load concentration until it is removed from the enclosed space via the outlet vents.

The temporal evolution of the viral load concentration field varies depending on location. We simulated coughing events at various positions and observed that the temporal evolution for locations that were not

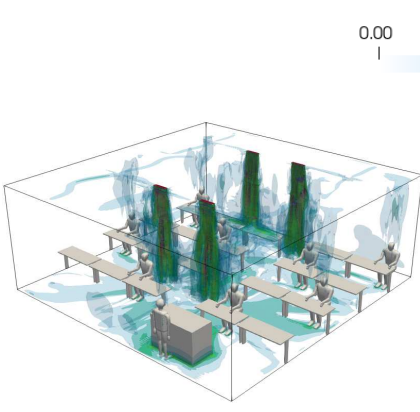

(a) Configuration 1

Velocity $(\mathrm{m} / \mathrm{s})$

$\begin{array}{lllllllll}0.00 & 0.200 & 0.400 & 0.600 & 0.800 & 1.00 & 1.20 & 1.40 & 1.60\end{array}$

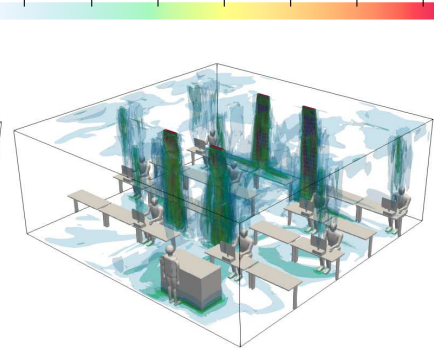

(b) Configuration 2

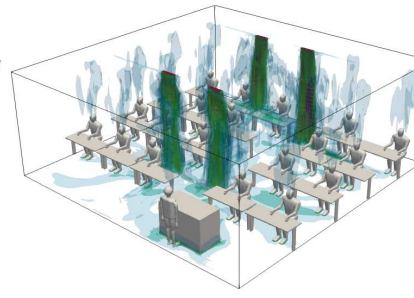

(c) Configuration 3

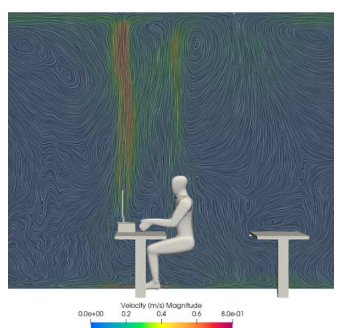

(d) Mid-plane streamlines of the flow around the objects

Figure 2: Visualization of the flow field field in a classroom for (a) Configuration 1: A classroom setting with 8 seated occupants and 1 standing occupant, (b) Configuration 2: A classroom setting with 8 seated occupants (each with a computer in front of them) and 1 standing occupant, (c) Configuration 3: A classroom setting with 20 seated occupants and 1 standing occupant and (d) streamlines representing the flow field around a mannequin and computer. 


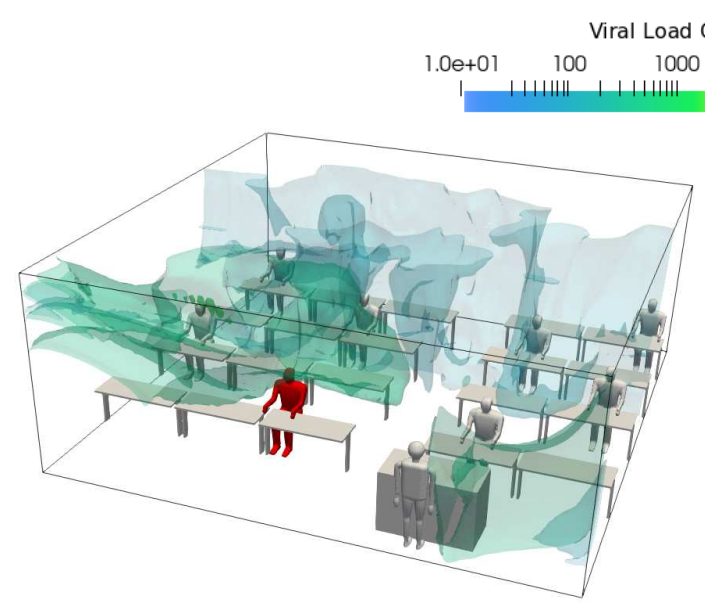

(a) Simulated cough by mannequin 2

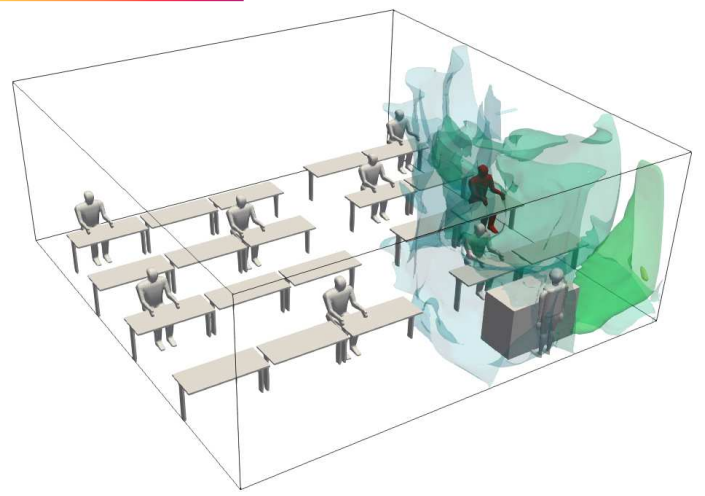

(b) Simulated cough by mannequin 3

Figure 3: Viral load concentration of the air 15 minutes after the simulated cough by the assigned mannequin (colored red).

close to (and right below) the inlet vents were similar regardless of location. Therefore, we focus our transmission risk assessment on two canonical locations: a simulated cough by an infected individual seated directly beneath an inlet vent (mannequin 2, Figure 3a) and one seated away from the inlet vent (mannequin 3, Figure 3b. Please refer to Figure S.4 for additional details and mannequin labels.

We observed that the virion-laden air dilutes more rapidly when the cough occurs in the vicinity of an inlet vent (Figure 3a), and the viral concentration remains higher in a more localized region when the cough occurs further away from an inlet (Figure 3b). This is due to mixing of the contaminated air with the inlet jet, resulting in a lower viral load concentration at the end of an air change cycle when the cough occurs closer to an inlet. This higher rate of dilution suggests a potentially lower risk of transmission.

From these results, we also observe that the virion-laden air is transported over a long distance when released near the inlet vents; thus, the contaminated air can potentially occupy a larger volume of the classroom space than when released further away from the inlet vents (Figure 3). Hence, occupants more than 6 feet away from the infected individual may still be susceptible to infection if the total number of inhaled particles exceeds the minimum infective dose (MID). To assess the transmission risk under this complicated interaction, we consider these two competing outcomes, the viral load concentration of the contaminated air and the volume of space occupied by the contaminated air.

\section{Transmission Risk Assessment}

We can quantify the transmission risk assessment in terms of the total number of virion particles inhaled by an individual at any given position in the classroom throughout the simulation time (see Equation (3)). The transmission risk is quantified and visualized on a horizontal plane at breathing height for seated individuals (see Figure S.2). We quantify the transmission risk for the five specific scenarios mentioned earlier. For a better visualisation of the transmission risk contours, black and white contour lines are added to the contour plots to represent 50 and 100 inhaled virion particles respectively.

As a baseline, a localized region with a high transmission risk is observed in the region in front of mannequin 3 for a simulated cough event (in Configuration 1). However, this localized region only directly affects one other occupant (mannequin 1) (Figure 4f). Otherwise, the high transmission region is confined to a space in the classroom that is not occupied. The black contour lines demarcate the region within which 50 or more virion particles will be inhaled ( $N \geq 50$ based on Equation (3); see Methods section). These regions represent the space with a higher risk of infection. In contrast, we found that every seated occupant in the classroom experiences (at worst) a moderate risk of infection due to a simulated cough by mannequin 2 (Figure 4a). Note that mannequin 2 is seated underneath an inlet vent. Although fewer occupants are at risk of infection due to an infected mannequin 3 (Figure $4 \mathrm{f}$ ), the affected individual would likely be more susceptible due to a higher count of inhaled virion particles than the exposure resulting from a cough by mannequin 2. 


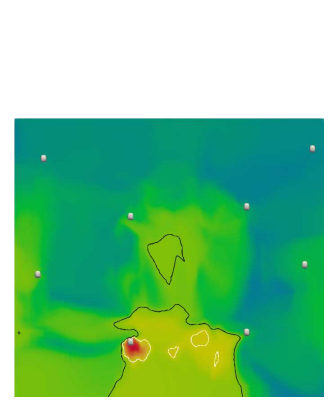

(a) Base case for comparison

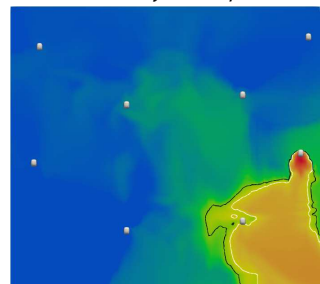

(f) Base case for comparison

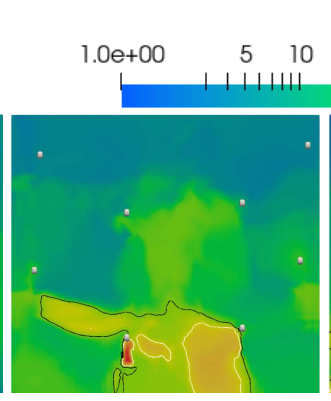

(b) With computers

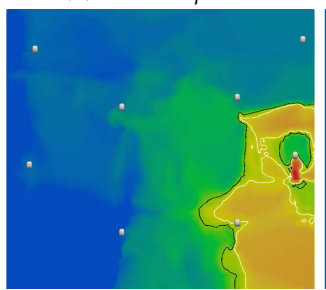

(g) With computers

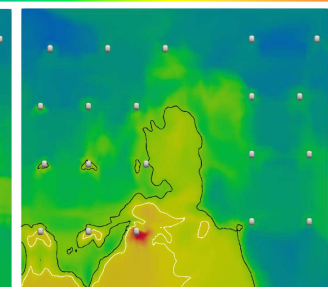

(c) Increased occupancy

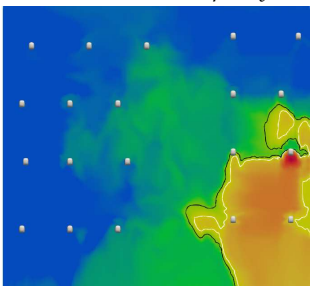

(h) Increased occupancy

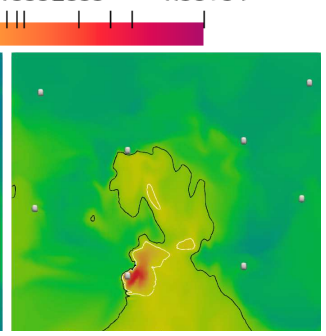

(d) With increased $\mathrm{ACH}$

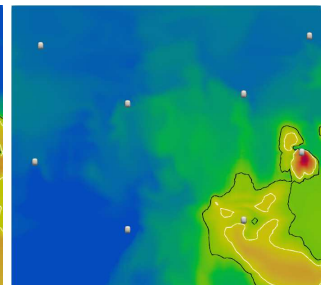

(i) With increased $\mathrm{ACH}$

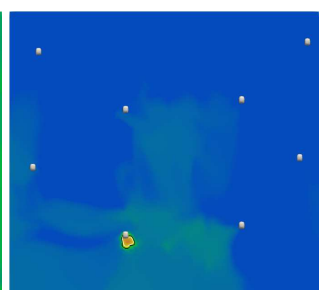

(e) All occupants masked

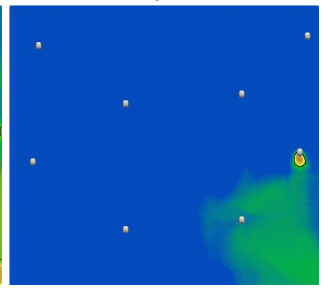

(j) All occupants masked

Figure 4: Top view of each case with the contour of transmission risk shown at the end of one air change cycle due to a simulated cough by mannequin 2 (top row) and mannequin 3 (bottom row).

Impact of Electronics: When an infected individual coughs, we expect the physical presence of electronic equipment (such as a computer monitor) to block the virion-laden air from reaching other individuals. Furthermore, the thermal plume generated by the electronics may impede pathogen transmission by transporting the pathogen-laden aerosols upward away from the breathing zone of other individuals. Hence, intuitively, we expect the transmission risk to be reduced.

Surprisingly, the addition of computers does not dissipate the risk of transmission. In fact, it expands the localized region that has a high risk of transmission (Figure $4 \mathrm{~b}$, Figure $4 \mathrm{~g}$ ) based on the risk transmission assessment for Configuration 2. This phenomenon is observed for both cases of simulated cough by mannequin 2 and mannequin 3 . The thermal plumes generated by the computers enhance the recirculation of the contaminated air and increase the region with higher transmission risk (Figure 2). In Configuration 2 (with reduced occupancy), the expanded region with high transmission risk does not further implicate any previously unaffected occupants since it is confined to a region of space that is unoccupied (Figure 4g). However, mannequin 1 will experience a greater risk of infection caused by a cough by mannequin 2 .

Impact of Increased Occupancy: Increased occupancy within an enclosed space decreases the proximity between individuals to $\approx 3$ feet and increases the ease of transmission of virion particles. It also introduces additional thermal plumes that alter the flow field of the classroom, complicating the risk assessment.

Based on the risk assessment conducted for Configuration 3, which represents a classroom setting with an occupancy of 20 students (Figure S.3c), we find that the region of transmission risk increases with occupancy Figure 4c, Figure 4h. In adherence to social distancing guidelines, a decrease in the occupancy of the classroom (from a class size of 20 in Configuration 3 to 8 in Configuration 2) decreases the risk slightly by increasing the distance the virion particles have to travel from their source (the infected cougher). However, social distancing guidelines still do not provide the occupants with sufficient protection against an infection risk in the presence of computers that redirect the flow, as shown in (Figure 4b, Figure 4g).

Impact of Increased Air Changes: An increase in air changes per hour $(\mathrm{ACH})$ for an enclosed space will increase the volume of purified air entering the classroom. We examined the effect of increased $\mathrm{ACH}$ on the transmission risk by increasing the $\mathrm{ACH}$ from 4 to 6 for Configuration 1 . In this case, we notice rapid dilution of the contaminated air due to a simulated cough at the higher $\mathrm{ACH}$. The higher ventilation with increased $\mathrm{ACH}$ reduces the size of the localized regions of transmission risk (Figure 4d, Figure 4i). 


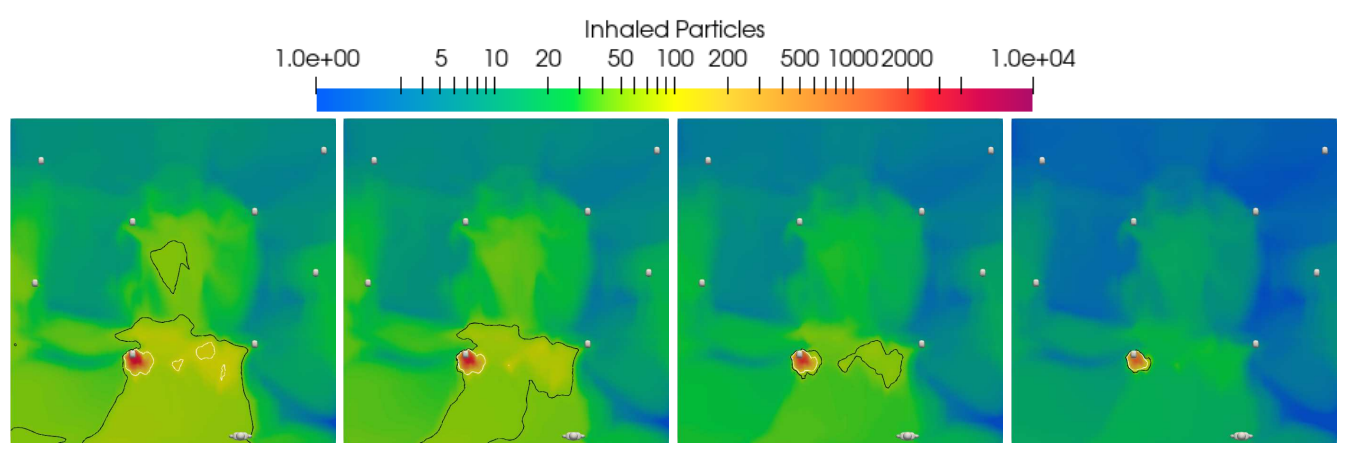

(a) Everyone is unmasked.

(b) Face masks with $18.8 \%$ IPE

(c) Face masks with $44.0 \%$ IPE.

(d) Face masks with $74.0 \%$ IPE.

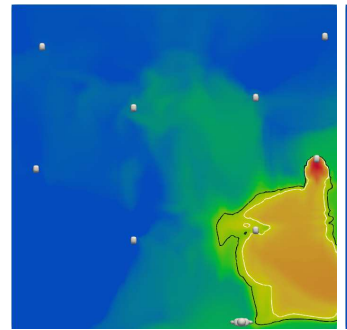

(e) Everyone is unmasked.

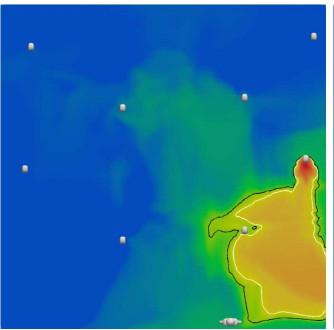

(f) Face masks with $18.8 \%$ IPE.

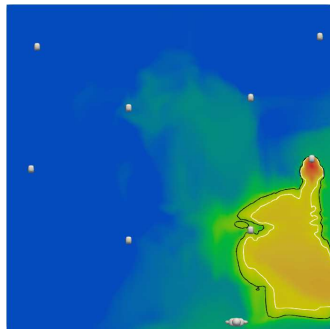

(g) Face masks with $44.0 \%$ IPE.

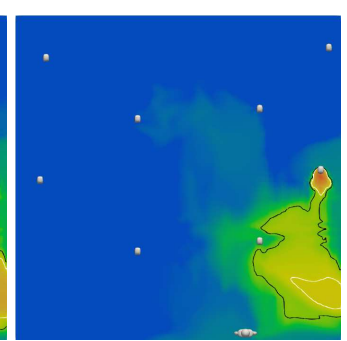

(h) Face masks with $74.0 \%$ IPE.

Figure 5: Comparison of the amount of protection each type of face masks provide users with against a simulated cough by unmasked mannequin 2 (top row) and mannequin 3 (bottom row).

Impact of Face Mask Usage: The use of face masks significantly reduces the viral load released by an infected individual during exhalation compared to other mitigation strategies. The flow resistance of face masks impedes the spread of the cough cloud from the individual. Hence, the cough cloud released by a masked individual differs in size and viral load density from an unmasked individual.

The impact of face masks on risk transmission is evaluated based on the classroom setting described in Configuration 1. In this configuration, all of the occupants are assumed to be wearing surgical masks. The surgical mask is capable of preventing $94 \%$ of the virion aerosols from being released by a masked cougher ${ }^{23}$, and it decreases the extent of the respective cough cloud to $35 \mathrm{~cm}$ (from $70 \mathrm{~cm}$ for a maskless cough) due to its flow resistance 24 . The inward protection efficiency (IPE) of the surgical mask worn by all occupants is assumed to be $18.81 \% 25$. This implies that the masked occupants inhale at most $81.19 \%$ of the virion-laden aerosols from the ambient air.

The use of face masks tremendously reduces the transmission risks such that no occupants have inhaled a significant quantity of virion particles (Figure 4e, Figure 4j) as compared to the case where a majority of the occupants in the class are at moderate risk of becoming infected because everyone is unmasked (Figure 4a. Figure 4f). Furthermore, the extent of the region of transmission risk is significantly reduced and confined to the space in front of the cougher's mouth. Thus, everyone wearing masks (even sub-optimally, as evidenced by the $18.8 \%$ inward protection efficiency) produces the most significant risk reduction to transmission.

While the transmission risk is minimal when every occupant is masked, this might not be the most prevalent scenario with the relaxation of the mask mandates. An unmasked asymptomatic carrier may endanger other individuals nearby, making them susceptible to infection. Hence, we investigate the protection offered by face masks to a masked individual from other unmasked and infected individuals.

Due to the gap between the face mask and the face, the IPE of the masks is significantly lower than the material filtration efficiency. The IPE of current existing surgical masks ranges from $18.8 \% 25$ to $44.0 \% 26$, to even $74 \%$. We next evaluate the impact on various IPE on risk profiles as shown in Figure 5. Most face masks provide users with sufficient protection against a cough by an unmasked mannequin 2 . However, mannequin 1 would still be susceptible to an infection due to cough by an unmasked mannequin 3 with the use of existing face masks with IPE between $18.8 \%$ to $44.0 \%$. This suggests a need to improve the mask design to get a better IPE that provides users with better protection against other unmasked individuals. 
Researchers have been proposing improved mask designs (as well as methods to achieve a snug fit) that result in higher IPE and can provide users with sufficient protection against an airborne transmission. Pan et al. ${ }^{27}$ have proposed an improved three-layer mask design that provides users with at least $74 \%$ IPE. The effectiveness of the improved face mask design is tested in our simulations with the results shown in Figure 5d and Figure 5h. These masks provide users with sufficient protection against other unmasked individuals. In most cases, with a high-efficiency mask, when worn correctly, the transmission risk to masked individuals is very low, even if the infected individual chooses to be unmasked (see additional data in Figure S.12, Figure S.13, Figure S.14|Figure S.14).

\section{Discussion}

Our results show that localized regions of high risk of transmission can exist in a classroom (and more generally indoor environments) even when occupants are well spaced apart and ventilation rates are increased. However, the risk of transmission is significantly reduced if the infected individual is masked. Even when an infected individual chooses to remain unmasked, proper masking-that ensures moderate infiltration efficiency (or inward protection efficiency)-by other individuals reduces the risk of transmission to them.

We next discuss simplifying assumptions made and their implications on our results.

- Distribution of initial cough cloud: A coughing event is initialized as a cough cloud represented by a cone-shaped region of homogeneously distributed virion concentration in front of the cougher's mouth. A small number of virion particles that lie outside the cough cloud are neglected (Figure S.1). Extending the cone-shaped region does not significantly change any of the results.

- Distribution of initial cough cloud under masked conditions: The viral shedding from leakages along the edges of the mask is neglected in the initialization of the cough droplets released by a masked cougher. A more accurate initial condition for the virion particles can improve the representation of the location of discrete virion particles outside of the cough cloud and in the leakage regions along the edges of the face mask. As before, slight changes to the initial cough cloud do not significantly change any of the conclusions.

- Scalar transport of virion particle density: We only consider particles of size less than $10 \mu \mathrm{m}$. We only consider the advective and diffusive transport of the virion particles (Equation 14). This equation does not account for the depletion of the viral load density in the contaminated air, perhaps due to the deactivation of the virus, depletion of virion particles when inhaled by the occupants, and the adhesion of virion particles to contacted surfaces. We also do not consider the impact of humidity on the distribution. In this context, our results can be considered conservative estimates of the transmission risk since relaxing these assumptions will reduce the virion concentration field and transmission risk.

- An assumption involved with the transmission risk assessment is that the viral load density remains relatively unchanged when inhaled by the occupants. This assumption is commonly adopted across the literature $7-9$.

Conclusions: We illustrate a computational approach that can automatically and efficiently evaluate transmission risk in geometrically complex indoor settings. This approach abstracts away the complexity of mesh generation and subsequent simulation and analysis, thus allowing straightforward deployment by end-users. The availability of such a personalized planning tool will enable educational institutions (K12 , community colleges, universities) and other entities to evaluate various in-person interaction scenarios comprehensively. Such a framework will help quantify and minimize the risk of infection. We illustrate the utility of the framework by evaluating risk for different seating, ventilation, and mask-wearing scenarios in a canonical classroom. Wearing masks significantly reduces the risk of transmission of the SARS-CoV-2 virus. More broadly, this framework for personalized transmission risk assessment may also be applied to other airborne transmittable pathogens based on the known information about the pathogen and its minimum infection dosage (MID) and can be useful in other critical care infrastructures such as hospitals (operation theaters, infectious wards, etc.). This work is a step in the path to democratization of complex simulation software to the broader scientific and application community. 


\section{Methods}

\subsection{Background}

The well-mixed room (WMR) model is a mathematical model that predicts the probability of airborne pathogen transmission within an enclosed space based on the time evolution of the airborne pathogen concentration. The WMR model computes the concentration of the airborne pathogen based on a simple mathematical model that relates the rate of change of the pathogen concentration to various parameters of the venue under consideration, such as the number of occupants, size of the room, shedding rate of virion particles from the infected host(s), rate of removal of pathogens from artificial/natural ventilation, rate of destabilization of the pathogen, etc. $\stackrel{\text { 28 }}{.}$. However, several assumptions are made in the WMR model, making it invalid under more complicated and real-life scenarios. Furthermore, the WMR model is incapable of computing the risk of pathogen transmission in the spatial domain.

Hence, most computational-based transmission risk assessment models focus on predicting the transport of the pathogen-laden aerosols by a computed flow field governed by the Navier-Stokes equations. In particular, most computational studies adopt the Eulerian-Lagrangian approach to compute the transport of respiratory droplet particles in air 2429 -31. In this approach, the fluid phase (air) is solved as a continuous phase, while the motion of the discrete phase (respiratory droplets) is computed based on the flow characteristics of the fluid phase and the properties of the discrete phase. Since the size of a respiratory droplet affects its transport properties (such as its molecular diffusion coefficient and drag coefficient ${ }^{32}$ ) and viral load 33 , information about the size distribution of the respiratory droplets is required for the EulerianLagrangian approaches. However, such information varies vastly across the highly cited literature 15$] 34,38$. This difference can be attributed to the different (and possibly wrong) techniques and assumptions involved with the measurements of the respiratory droplets 39 . Using an unsuitable droplet size distribution in an Eulerian-Lagrangian approach would imply an error in the predicted transport path of respiratory droplets and, therefore, the assessment of the transmission risk. Furthermore, since approximately $O\left(10^{5}\right)$ airborne particles may be released in a single $\operatorname{cough} \underline{40}$, it may be computationally expensive to use the Eulerian-Lagrangian approach to predict the particle transport, especially in a complex flow field.

In the alternative Eulerian-Eulerian approach, the fluid phase and the airborne respiratory virion particles are both treated as continuous phases in the solution of their respective governing equations. This approach has been used infrequently for the prediction of pathogen transmission for SARS-CoV-2 so far. The Eulerian-Eulerian approach provides users with a lowered computational cost, which is beneficial for parameter studies where multiple scenarios need to be simulated to analyze the effect of the various factors on the variable of interest.

The discretization of the computational domain for most SARS-CoV-2 transmission simulations is performed with an unstructured tetrahedral body-fitted mesh. Ideally, structured or hexahedral body-fitted meshes would be desired for the solution of a discretized domain due to the generally lowered cell count, better convergence rate, and lowered computational cost compared to tetrahedral meshes ${ }^{41}$. However, the generation of structured or hexahedral meshes requires significantly more time and effort than that of unstructured mesh generation, especially for a computational domain with geometric complexities ${ }^{42}$. Furthermore, body-fitted meshes (both unstructured and hexahedral) require a certain degree of manual effort to design a discretized computational domain that conforms to the surface of the complicated geometries involved ${ }^{43}$. Hence, discretizing a computational domain with a body-fitted mesh can be computationally expensive and cumbersome, especially when multiple geometrically different computational domains have to be adopted for a parameter study.

Tree-based adaptive mesh generation would be a suitable approach for the creation of the meshes in a parameter study 1920 . This mesh generation approach involves immersing the geometries into a background mesh before carving out the mesh that resides within the geometries. The carved-out space in the background mesh represents the region occupied by the geometries, while the effect of the geometries' surfaces is represented using the voxelized boundaries. In contrast to unstructured background meshes, the octreebased parallel adaptive mesh is capable of scalability at extreme scales. It also ensures the generation of a high-quality background mesh with a uniform aspect ratio. This method relaxes the requirements for the mesh quality along the boundaries of the immersed geometries. Also, it provides users with a swift method 


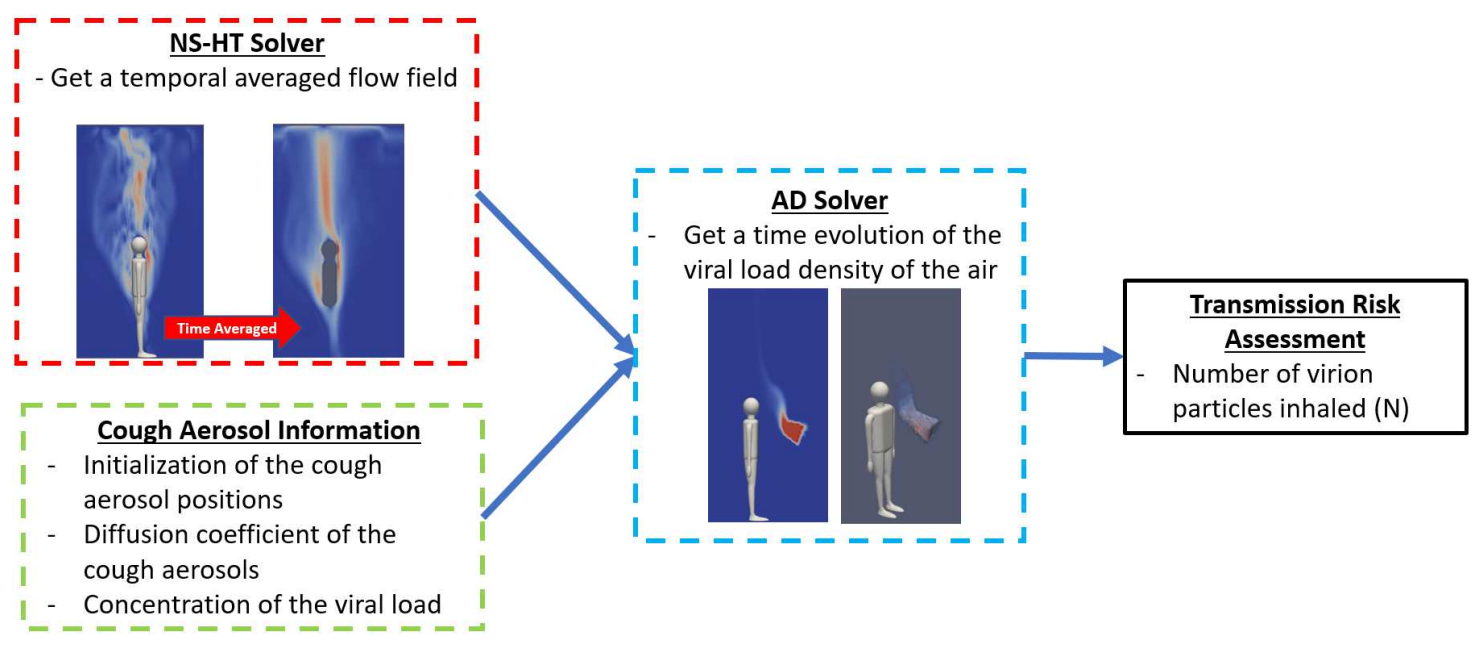

Figure 6: Simulation workflow to compute the transmission risk.

of generating a discretized computational domain, which is essential for the generation of meshes for the multiple geometrically different computational domains required in a parameter study.

In terms of quantifying transmission risk, we observed a generally similar assessment method across the literature. Some authors quantify the risk of transmission in terms of the amount of pathogen released by the carrier ${ }^{29 \mid 44}$. In contrast, others quantify the risk in terms of the number of virion particles being inhaled by susceptible persons $^{7-9} 9$. The release of virion particles from a carrier does not necessarily result in a transmission unless others inhale the virion particles. However, the inhalation of virion particles does not equate to certainty in disease transmission due to the minimum infective dose (MID) required for susceptible individuals to become infected. Based on a study of the virion exposures for multiple superspreading events, it has been hypothesized that the MID for SARS-CoV-2 infection in susceptible people is 50 quanta 28 . Hence, the inhalation of virion particles below the speculated MID into the respiratory system is unlikely to cause an infection.

Since it has been postulated that duration of exposure to a pathogen (in addition to the physical distance from a disease carrier ${ }^{\sqrt{5}}$ ) is another contributing factor to disease transmission ${ }^{8}$, the transmission risk assessment should also account for the exposure time. We can fulfill the consideration of exposure time in the transmission risk assessment by relating the risk of transmission to the cumulative sum of the viral load density at the respective position within the considered domain. This can indicate the total number of virion particles inhaled by an occupant at that position over the simulated duration. We can then compare the cumulative amount of virion particles inhaled at a location with the MID to gauge if a susceptible individual is prone to infection.

\subsection{Computational Model}

We adopt an Eulerian-Eulerian-based Finite Element Method (FEM) approach with linear basis functions and variational multiscale (VMS) ${ }^{21446}$ framework to discretize and solve the governing equations for the flow and transport of the virion particles. The momentum and heat transfer equations are solved concurrently with an in-house Navier-Stokes Heat Transfer (NS-HT) solver to compute the time evolution of the flow field within the classroom. A temporally averaged flow field in the classroom is then obtained across multiple time steps after the flow field reaches a statistically steady state. The time-averaged flow field is then used as an advection field to compute the transport of the virion-laden aerosols in the advectiondiffusion (AD) solver. The simulation workflow is depicted in a schematic shown in Figure 6 Please refer to the Supplement for details on the Variational Multiscale (VMS) formulation for the flow physics.

Information about the virion-laden aerosols (such as the initialization of their position due to a cough, viral load density, and diffusion coefficient) is also required as inputs to the AD solver. The virion-laden aerosols are modeled as a continuum and considered a passive scalar in the AD solver. The backward differentiation formula 2 (BDF2) is used as the time-stepping scheme in the solution of the governing equations. 

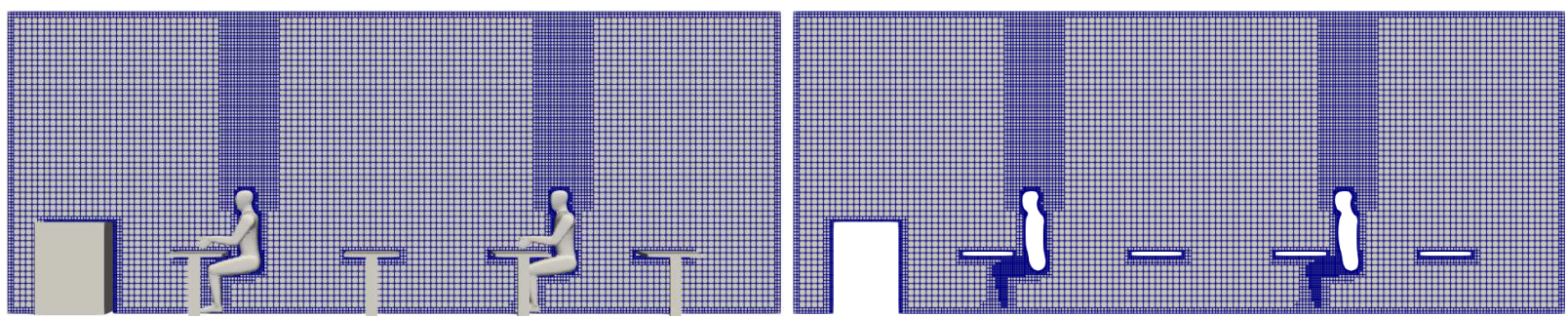

Figure 7: Various solid objects (mannequins, desks, tables, computers) are placed in the computational domain, and the background mesh is carved out and successively refined. This produces an incomplete octree-based mesh of the computational domain (right).

Information about the viral load density of the air, obtained from the solution of the AD solver, is then post-processed to compute the number of virion particles that would be inhaled at every location within the classroom $(\mathrm{N})$. The magnitude of $\mathrm{N}$ at every location within the classroom is compared with the MID for the transmission risk assessment.

We use CAD models (STL files) of desks, chairs, and monitors to realistically represent various solid (and slender) objects that can impact the flow patterns. We utilize STL files of mannequins to represent human subjects. These STL files allow straightforward reconfiguration and exploration of various scenarios. We consider a fall semester operating condition and take into account the heat flux from both humans and electronic equipment.

\subsection{Octree-Based Adaptive Discretization}

Conventional body-fitted meshing is both case-specific and labor-intensive to generate. In this study, several geometrically different computational domains with different mesh requirements are required due to the presence of varying objects (such as additional computers or mannequins) placed within the computational domain. Hence, we use an incomplete octree-based adaptive discretization approach for the mesh generation in the computational domains used in this study 19 .

The generation of the incomplete octree-based mesh begins with creating a cuboid background mesh composed of cubic elements. Then, a water-tight manifold of the bodies in the computational domain (such as the mannequins, tables, and computers) is immersed in the background mesh. Since the elements of the background mesh in the interior of the manifold are not involved in the numerical solutions of the governing equations, they are removed to reduce the memory footprint required, generating a "carved out" domain in the background mesh. Then, the respective strong boundary conditions for the various governing equations are applied onto the surface of the carved-out region, as shown in Figure 7. This mesh generation process is fully automated, and no manual geometry or mesh cleanup is required.

The degree of mesh density of the cubic elements within the octree-based grids is defined by level. Each cubic element has a length of $\max \left(\right.$ domain) $/ 2^{\text {level }}$, where $\max ($ domain $)$ is the length of the longest edge of the computational domain. The degree of mesh density may be increased in specified regions and near the carved-out domains by subdividing the coarser elements in each axis.

The octree-based adaptive discretization provides the simulations with cubic elements of good mesh quality. It is also efficient in generating meshes for the geometrically different computational domains. This alleviates the time-consuming and laborious process of generating a mesh manually.

\subsection{Representation of the Region Occupied by Respiratory Aerosols}

A turbulent cloud of buoyant gas with suspended respiratory particles is exhaled from the mouth when an individual coughs. This cough cloud can be represented by a cone-shaped region diverging outward from the mouth of a cougher. The size, shape, and orientation of the cough cloud can be defined based on parameters such as the entrainment coefficient $\left(\alpha^{\prime}\right)$, coughing angle $(\theta)$, and size of the mouth (see Figure S.1. in Supplement) 10 .

For this computational study, we assume that the airborne virion aerosols released from a cough are homogeneously distributed throughout the cough cloud before they are subjected to advective and diffusive transport in the $\mathrm{AD}$ solver. The region occupied by the cough cloud is defined with a nondimensional viral 
load density of $C^{*}=1$. In contrast, the reference concentration can be defined based on the known viral load density of the cough cloud.

A single cough from an infected host may release as many as $1.23 \times 10^{5}$ copies of SARS-CoV-2 viruses that can remain airborne after 10 seconds 47 ; $94 \%$ of these can be effectively blocked by a surgical face mask $^{23}$. The flow resistance of face masks also impedes the spread of the cough cloud from the infected individual into the surroundings 48 . The cough cloud released by the infected individual can be defined using an entrainment coefficient of $\alpha^{\prime}=0.2116$ radians, downward coughing angle of $\theta=23.9^{\circ}$, and a circular mouth area of $3.4 \mathrm{~cm}^{2} 10$, with an extent of $70 \mathrm{~cm}$ for an unmasked cougher and $35 \mathrm{~cm}$ for a masked cougher 24 .

\subsection{Metric for Risk Assessment}

The risk of infection in the classroom can be quantified by the amount of virion particles inhaled by susceptible individuals at various locations within the classroom relative to that of the SARS-CoV-2 carrier. The number of virion particles drawn into the body with one inhalation breath can be calculated based on the following:

$$
N=C(t) \times V_{b}
$$

where $C(t)$ is the concentration of virion particles in the air being inhaled and $V_{b}$ is the volume of one inhalation. The magnitude of $V_{b}$ can be defined as $500 \mathrm{ml}$ based on tidal breathing 49 .

The total amount of inhaled virus-laden particles that are cumulatively inhaled at a position over a duration $T$ can be calculated based on

$$
N=\int_{0}^{T} C(t) \dot{V}_{b} d t
$$

where $\dot{V}_{b}$ is the continuous inhalation rate for humans with an average rate of $1.33 \times 10^{-4} \mathrm{~m}^{3} / \mathrm{s}$, based on 12 to 16 breaths per minute ${ }^{50}$.

The total number of inhaled particles is computed over a duration of 15 minutes, which is the duration for one complete air change within the classroom due to mixing ventilation based on $4 \mathrm{ACH}$. The total amount of virion particles inhaled by a susceptible person (Equation (2) can be computed from a scaled value of the cumulative sum of the viral load concentration across the various continuous time steps given by

$$
N \approx \sum\left[C(t) \dot{V}_{b} \Delta t\right]=\dot{V}_{b} \times \Delta t \times \sum\left[C^{*}(t)\right] .
$$

Although the MID of SARS-CoV-2 is currently unknown, it is hypothesized to be approximately 50 based on a well-mixed room modeling of 20 super spreading events 28 . Hence, regions with contours of inhaled virion particles with a count greater than 50 would imply that susceptible individuals risk infection if their breathing organs (mouth or nose) reside within these regions. A horizontal slice of the contour for the inhaled particle count is taken at the height of $1.25 \mathrm{~m}$ to evaluate the transmission risk of the seated occupants. This height corresponds to the location of the breathing organs of the mannequins (see Figure S.2 in Supplement). Based on this risk assessment metric, we can identify the regions where occupants can be susceptible to SARS-CoV-2 viral infection. 


\section{References}

[1] Centers for Disease Control (CDC). How COVID-19 spreads, 2020. URL https://www.cdc.gov/coronavirus/ 2019-ncov/prevent-getting-sick/how-covid-spreads.html

[2] World Heath Organization (WHO). Advice for the public on COVID-19, 2021. URL https://www. who.int/ emergencies/diseases/novel-coronavirus-2019/advice-for-public

[3] Lea Hamner. High SARS-CoV-2 attack rate following exposure at a choir practice-skagit county, washington, march 2020. MMWR. Morbidity and mortality weekly report, 69, 2020.

[4] Shelly L Miller, William W Nazaroff, Jose L Jimenez, Atze Boerstra, Giorgio Buonanno, Stephanie J Dancer, Jarek Kurnitski, Linsey C Marr, Lidia Morawska, and Catherine Noakes. Transmission of SARS-CoV-2 by inhalation of respiratory aerosol in the skagit valley chorale superspreading event. Indoor air, 31(2):314-323, 2021.

[5] Neeltje van Doremalen, Trenton Bushmaker, Dylan H. Morris, Myndi G. Holbrook, Amandine Gamble, Brandi N. Williamson, Azaibi Tamin, Jennifer L. Harcourt, Natalie J. Thornburg, Susan I. Gerber, and et al. Aerosol and surface stability of SARS-CoV-2 as compared with sars-cov-1. New England Journal of Medicine, 382(16):1564-1567, 2020. doi: $10.1056 /$ nejmc2004973.

[6] Nan Zhang, Xuguang Chen, Wei Jia, Tianyi Jin, Shenglan Xiao, Wenzhao Chen, Jian Hang, Cuiyun Ou, Hao Lei, Hua Qian, et al. Evidence for lack of transmission by close contact and surface touch in a restaurant outbreak of COVID-19. Journal of Infection, 2021.

[7] Siyao Shao, Dezhi Zhou, Ruichen He, Jiaqi Li, Shufan Zou, Kevin Mallery, Santosh Kumar, Suo Yang, and Jiarong Hong. Risk assessment of airborne transmission of COVID-19 by asymptomatic individuals under different practical settings. Journal of Aerosol Science, 151:105661, 2021. ISSN 0021-8502. doi: https://doi.org/10.1016/j.jaerosci. 2020.105661. URL/https://www. sciencedirect.com/science/article/pii/S0021850220301476.

[8] Ville Vuorinen, Mia Aarnio, Mikko Alava, Ville Alopaeus, Nina Atanasova, Mikko Auvinen, Nallannan Balasubramanian, Hadi Bordbar, Panu Erästö, Rafael Grande, and et al. Modelling aerosol transport and virus exposure with numerical simulations in relation to SARS-CoV-2 transmission by inhalation indoors. Safety Science, 130: 104866, 2020. doi: 10.1016/j.ssci.2020.104866.

[9] Zhihang Zhang, Taehoon Han, Hee Yoo Kwang, Jesse Capecelatro, André L. Boehman, and Kevin Maki. Disease transmission through expiratory aerosols on an urban bus. Physics of Fluids, 33(1), 2021. doi: 10.1063/5.0037452.

[10] Lydia Bourouiba, Eline Dehandschoewercker, and John W. M. Bush. Violent expiratory events: on coughing and sneezing. Journal of Fluid Mechanics, 745:537-563, 2014. doi: 10.1017/jfm.2014.88.

[11] Lydia Bourouiba. The fluid dynamics of disease transmission. Annual Review of Fluid Mechanics, 53(1):473-508, 2021. doi: 10.1146/annurev-fluid-060220-113712. URL https://doi.org/10.1146/ annurev-fluid-060220-113712

[12] Siddhartha Verma, Manhar Dhanak, and John Frankenfield. Visualizing the effectiveness of face masks in obstructing respiratory jets. Physics of Fluids, 32(6):061708, June 2020. doi: 10.1063/5.0016018. URL https: //doi.org/10.1063/5.0016018

[13] Rajat Mittal, Rui Ni, and Jung-Hee Seo. The flow physics of COVID-19. Journal of Fluid Mechanics, 894:F2, 2020. doi: $10.1017 / \mathrm{jfm} .2020 .330$.

[14] J.W. Tang, W.P. Bahnfleth, P.M. Bluyssen, G. Buonanno, J.L. Jimenez, J. Kurnitski, Y. Li, S. Miller, C. Sekhar, L. Morawska, and et al. Dismantling myths on the airborne transmission of severe acute respiratory syndrome coronavirus-2 (SARS-CoV-2). Journal of Hospital Infection, 110:89-96, 2021. doi: 10.1016/j.jhin.2020.12.022.

[15] C.Y.H. Chao, M.P. Wan, L. Morawska, G.R. Johnson, Z.D. Ristovski, M. Hargreaves, K. Mengersen, S. Corbett, Y. Li, X. Xie, and D. Katoshevski. Characterization of expiration air jets and droplet size distributions immediately at the mouth opening. Journal of Aerosol Science, 40(2):122-133, 2009. ISSN 0021-8502. doi: https://doi.org/10. 1016/j.jaerosci.2008.10.003. URL https://www. sciencedirect. com/science/article/pii/S0021850208001882.

[16] Mark Nicas, William W. Nazaroff, and Alan Hubbard. Toward understanding the risk of secondary airborne infection: Emission of respirable pathogens. Journal of Occupational and Environmental Hygiene, 2(3):143-154, March 2005. doi: 10.1080/15459620590918466. URL https://doi.org/10.1080/15459620590918466

[17] Jeffrey Slotnick, Abdollah Khodadoust, Juan Alonso, David Darmofal, William Gropp, Elizabeth Lurie, and Dimitri Mavriplis. CFD vision 2030 study: A path to revolutionary computational aerosciences, 2014. 
[18] F. Xu, D. Schillinger, D. Kamensky, V. Varduhn, C. Wang, and M.-C. Hsu. The tetrahedral finite cell method for fluids: Immersogeometric analysis of turbulent flow around complex geometries. Computers E Fluids, 141:135-154, 2016.

[19] Kumar Saurabh, Masado Ishii, Milinda Fernando, Boshun Gao, Kendrick Tan, Ming-Chen Hsu, Adarsh Krishnamurthy, Hari Sundar, and Baskar Ganapathysubramanian. Scalable adaptive PDE solvers in arbitrary domains. arXiv preprint arXiv:2108.03757, 2021. To be published in the proceedings of Super Computing (SC) 2021.

[20] Kumar Saurabh, Boshun Gao, Milinda Fernando, Songzhe Xu, Makrand A Khanwale, Biswajit Khara, Ming-Chen Hsu, Adarsh Krishnamurthy, Hari Sundar, and Baskar Ganapathysubramanian. Industrial scale Large Eddy Simulations with adaptive octree meshes using immersogeometric analysis. Computers $\mathcal{E}$ Mathematics with Applications, 97:28-44, 2021.

[21] Songzhe $\mathrm{Xu}$, Boshun Gao, Ming-Chen Hsu, and Baskar Ganapathysubramanian. A residual-based variational multiscale method with weak imposition of boundary conditions for buoyancy-driven flows. Computer Methods in Applied Mechanics and Engineering, 352:345-368, 2019.

[22] Joseph G. Allen and Andrew M. Ibrahim. Indoor air changes and potential implications for SARS-CoV-2 transmission. JAMA, 325(20):2112, 2021. doi: 10.1001/jama.2021.5053.

[23] Liqiao Li, Muchuan Niu, and Yifang Zhu. Assessing the effectiveness of using various face coverings to mitigate the transport of airborne particles produced by coughing indoors. Aerosol Science and Technology, 55(3):332-339, 2020. doi: 10.1080/02786826.2020.1846679.

[24] Talib Dbouk and Dimitris Drikakis. On respiratory droplets and face masks. Physics of Fluids, 32(6), Jun 2020. doi: 10.1063/5.0015044.

[25] Mervin Zhao, Lei Liao, Wang Xiao, Xuanze Yu, Haotian Wang, Qiqi Wang, Ying Ling Lin, F. Selcen Kilinc-Balci, Amy Price, Larry Chu, and et al. Household materials selection for homemade cloth face coverings and their filtration efficiency enhancement with triboelectric charging. Nano Letters, 20(7):5544-5552, 2020. doi: 10.1021/acs. nanolett.0c02211.

[26] Abhiteja Konda, Abhinav Prakash, Gregory A Moss, Michael Schmoldt, Gregory D Grant, and Supratik Guha. Aerosol filtration efficiency of common fabrics used in respiratory cloth masks. ACS nano, 14(5):6339-6347, 2020.

[27] Jin Pan, Charbel Harb, Weinan Leng, and Linsey C. Marr. Inward and outward effectiveness of cloth masks, a surgical mask, and a face shield. Aerosol Science and Technology, 55(6):718-733, 2021. doi: 10.1080/02786826.2021. 1890687.

[28] John M. Kolinski and Tobias M. Schneider. Superspreading events suggest aerosol transmission of SARS-CoV-2 by accumulation in enclosed spaces. Phys. Rev. E, 103:033109, Mar 2021. doi: 10.1103/PhysRevE.103.033109. URL https://link.aps.org/doi/10.1103/PhysRevE.103.033109

[29] Dongjie Jia, Jonathan Lee Baker, Anaïs Rameau, and Mahdi Esmaily. Simulation of a vacuum helmet to contain pathogen-bearing droplets in dental and otolaryngologic outpatient interventions. Physics of Fluids, 33(1):013307, 2021. doi: $10.1063 / 5.0036749$.

[30] Sai Ranjeet Narayanan and Suo Yang. Airborne transmission of virus-laden aerosols inside a music classroom: Effects of portable purifiers and aerosol injection rates. Physics of Fluids, 33(3):033307, 2021. doi: 10.1063/5.0042474.

[31] Hongying Li, Fong Yew Leong, George Xu, Zhengwei Ge, Chang Wei Kang, and Keng Hui Lim. Dispersion of evaporating cough droplets in tropical outdoor environment. Physics of Fluids, 32(11):113301, Nov 2020. doi: $10.1063 / 5.0026360$.

[32] Vladimir P. Zhdanov and Bengt Kasemo. Virions and respiratory droplets in air: Diffusion, drift, and contact with the epithelium. Biosystems, 198:104241, 2020. ISSN 0303-2647. doi: https://doi.org/10.1016/j.biosystems.2020. 104241. URL https://www.sciencedirect.com/science/article/pii/S0303264720301295.

[33] Kristen K Coleman, Douglas Jie Wen Tay, Kai Sen Tan, Sean Wei Xiang Ong, Ming Hui Koh, Yi Qing Chin, Haziq Nasir, Tze Minn Mak, Justin Jang Hann Chu, Donald K Milton, et al. Viral load of SARS-CoV-2 in respiratory aerosols emitted by COVID-19 patients while breathing, talking, and singing. medRxiv, 2021.

[34] Mohamed Abuhegazy, Khaled Talaat, Osman Anderoglu, and Svetlana V Poroseva. Numerical investigation of aerosol transport in a classroom with relevance to COVID-19. Physics of Fluids, 32(10):103311, 2020. 
[35] J. P. Duguid. The size and the duration of air-carriage of respiratory droplets and droplet-nuclei. Epidemiology and Infection, 44(6):471-479, 1946. doi: 10.1017/s0022172400019288.

[36] L. Morawska, G.R. Johnson, Z.D. Ristovski, M. Hargreaves, K. Mengersen, S. Corbett, C.Y.H. Chao, Y. Li, and D. Katoshevski. Size distribution and sites of origin of droplets expelled from the human respiratory tract during expiratory activities. Journal of Aerosol Science, 40(3):256-269, 2009. ISSN 0021-8502. doi: https://doi.org/10.1016/ j.jaerosci.2008.11.002. URL https://www.sciencedirect.com/science/article/pii/S0021850208002036.

[37] Xiaojian Xie, Yuguo Li, Hequan Sun, and Li Liu. Exhaled droplets due to talking and coughing. Journal of The Royal Society Interface, 6, 2009. doi: 10.1098/rsif.2009.0388.focus.

[38] Shinhao Yang, Grace Lee, Cheng-Min Chen, Chih-Cheng Wu, and Kuo-Pin Yu. The size and concentration of droplets generated by coughing in human subjects. Journal of Aerosol Medicine, 20(4):484-494, 2007. doi: 10.1089/ jam.2007.0610.

[39] G.R. Johnson, L. Morawska, Z.D. Ristovski, M. Hargreaves, K. Mengersen, C.Y.H. Chao, M.P. Wan, Y. Li, X. Xie, D. Katoshevski, and S. Corbett. Modality of human expired aerosol size distributions. Journal of Aerosol Science, 42(12):839-851, 2011. ISSN 0021-8502. doi: https://doi.org/10.1016/j.jaerosci.2011.07.009. URL https://www. sciencedirect.com/science/article/pii/S0021850211001200.

[40] William G Lindsley, Terri A Pearce, Judith B Hudnall, Kristina A Davis, Stephen M Davis, Melanie A Fisher, Rashida Khakoo, Jan E Palmer, Karen E Clark, Ismail Celik, et al. Quantity and size distribution of coughgenerated aerosol particles produced by influenza patients during and after illness. Journal of occupational and environmental hygiene, 9(7):443-449, 2012.

[41] Yongjie Jessica Zhang. Geometric Modeling and Mesh Generation from Scanned Images. CRC Press, Boca Raton, 2016.

[42] Marshall Bern and Paul Plassmann. Mesh generation. Handbook of Computational Geometry, page 291-332, 2000. doi: 10.1016/b978-044482537-7/50007-3.

[43] Michael J Aftosmis. Solution adaptive Cartesian grid methods for aerodynamic flows with complex geometries. von Karman Institute for Fluid Dynamics Computational Fluid Dynamics Lecture Series, Mar 1997.

[44] Martin Z. Bazant and John W. Bush. A guideline to limit indoor airborne transmission of covid-19. Proceedings of the National Academy of Sciences, 118(17), 2021. doi: 10.1073/pnas.2018995118.

[45] M. Elizabeth Halloran, Ira M. Longini, and Claudio Struchiner. Design and analysis of vaccine studies. Statistics for Biology and Health, 2010. doi: 10.1007/978-0-387-68636-3.

[46] Y. Bazilevs, V.M. Calo, J.A. Cottrell, T.J.R. Hughes, A. Reali, and G. Scovazzi. Variational multiscale residual-based turbulence modeling for large eddy simulation of incompressible flows. Computer Methods in Applied Mechanics and Engineering, 197:173-201, 2007.

[47] Yang Wang, Guang Xu, and Yue-Wern Huang. Modeling the load of SARS-CoV-2 virus in human expelled particles during coughing and speaking. PLOS ONE, 15(10), Oct 2020. doi: 10.1371/journal.pone.0241539.

[48] Christian J. Kähler and Rainer Hain. Fundamental protective mechanisms of face masks against droplet infections. Journal of Aerosol Science, 148:105617, 2020. ISSN 0021-8502. doi: https://doi.org/10.1016/j.jaerosci.2020.105617. URL https://www.sciencedirect.com/science/article/pii/S0021850220301063

[49] Sasha Hallett. Physiology, Tidal volume, Jun 2020. URL https://www.ncbi.nlm.nih.gov/books/NBK482502/

[50] Kim E. Barrett, Susan M. Barman, Scott Boitano, and Heddwen L. Brooks. Ganong's review of medical physiology. McGraw-Hill, 24 edition, 2013. 


\section{Supplementary Files}

This is a list of supplementary files associated with this preprint. Click to download.

- SI.pdf 